\title{
Analytical Method of Human Systemic and Global Circulation
}

\author{
Francis Egenti Nzerem ${ }^{1}$ and Eucharia C. Nwachukwu ${ }^{2}$
}

\begin{abstract}
The human circulatory system is one of the admirable rhythms of nature. The heart and the vasculature are constitutive structures. The vasculature consists of arterial and venous appurtenances which are arranged in an idealized network capable of enhancing circulation. The crux of this study is the representation of the cardiovascular system as a network in which electrical constraints apply. As a network, the system is amenable to graph analytic treatment; as edge-nodal parameters ensue, topological constraints apply. In virtue of cardiac autorhythmicity, electrical impulses are driven through the vessels to the body cells. As a rule, the vessels must elicit a modicum of resistance. This work weaponized the elements of graph theory and electrical properties of the heart in elucidating the flow mechanism associated with the cardio-vascular system. The voltage drop across the connecting vessels (idealized as wires) was carefully depicted and analyzed by the method of matrices. When the cardiac function is within physiological definition a vascular compartment may be a liability in the event of poor circulation. Therefore the knowledge of vascular resistive capacities, which this work portrayed, is a sine-qua-non to the assessment of flow integrity of the system under consideration.
\end{abstract}

MSC 2010 No.: 05C21, 92C42, 92B25.

Keywords: Cardiovascular, Network, Matrices, Flow, Circuit, Edges and Nodes, Wave propagation, Bifurcation.

1 Department of Mathematics and Statistics, Faculty of Sciences University of Port Harcourt Choba, Nigeria.

2 Department of Mathematics and Statistics, Faculty of Sciences University of Port Harcourt Choba, Nigeria.

Article Info: Received: September 28, 2021. Revised: October 27, 2021.

Published online: November 26, 2021. 


\section{Introduction}

The cardiovascular system (CVS) or circulatory system transports nutrients, metabolic products, and respiratory gases all through living cells, allowing vivacity among the various tissues. Circulation involves the intake the transmission_of metabolic materials all through the organism, and the delivery of harmful byproducts to the environment. The heart (pump) and a network of blood vessels are the appurtenances of circulation. The circulatory system is divided into two parts: the pulmonary and systemic circulations. The pulmonary circulation system defines the network of blood vessels from the right heart to the lungs and back to the left heart. It is known as the small circulation as it is only between the heart and the lungs. The large (systemic) circulation takes oxygenated blood from the left ventricle through the aorta to the different organs through the arterial system and eventually takes it back to the right atrium through the venous system and the vena cava. At a peak pressure of about $4 \mathrm{kPa}$ blood is pumped from the right ventricle through the pulmonary artery to the lungs. Venous blood which enters the pulmonary system becomes oxygenated and returns to the left heart atrium. The blood vessels (vasculature) consist of five categories: arteries and arterioles (making up the arterial system), veins and venules (the venous system), and capillaries (the smallest blood vessels, connecting arterioles and venules through networks within organs and tissues). Arteries are defined as 'bifurcating' vessels. A typical case is an aorta that branches off into smaller arteries and arterioles. On the other hand, veins are defined as 'converging' vessels, for example, venules and veins connect to return blood to the heart through the major veins (such as the superior and inferior venae cavae), Jarvis [1]. Capillaries are in close communication with the tissues, supplying nutrients and eliminating wastes through their reedy walls at a cellular level. Details of the circulatory system may be found in physiology pieces of literature such as Matthews et al [2] Jarvis and Saman [3], and Jarvis [1].

In recent times, mathematical models and numerical analysis have made remarkable progress in the explanation of circulatory system functionality. Several treatments on this functionality are compartmental. Smith et al. [4] propose a model of blood circulation in the coronary network. They treated major vessels as a connected onedimensional network, and arterioles, capillaries, and venules, are treated as lumped components that are connected to the network of vessels. Ruan et al. [5] studied blood circulation in the brain and Johannes et al. [6] showed that cerebral blood flow may well be modelled as fluid flow driven through a network of resistors by pressure gradients. In their contribution, Ketan and Bhathawala [7] developed a model of the human cardiovascular system using lumped parameter method. By relying on the assertion that every closed fluid system has a similitude of an electrical circuit, they modelled the cardiovascular system as analogous to the electrical circuits. The lumped parameter model approach had been a choice method in detailing various parts of the cardiovascular systems Rideout and Katra [8], Rideout and Dick [9], Snyder and Rideout [10], Liang et al. [11], Formaggia et al. [12]. In every case, the fluid and structure synergy must adhere to acceptable 
compatibility conditions along some characteristics as may be seen later as well as conditions of mass conservation, all of which are boundary conditions Blanco and Feij oo [13], and Alastruey et al. [14].

This work considered flow through some organs of the human body, to wit the heart, lungs, digestive tract, liver, kidney, trunks and legs, head and arms. It afforded a novel approach to describing the human circulatory system utilizing a graphtheoretic network. The network was treated as a hypothetical electrical analogue with topological constraints. This was done on the basis that the cardiac cells are electrical current sources Nzerem and Ugorji [15]. Topological constraints arising from the, now electrical, network configuration were depicted and the resistance of the vasculature provoked by flow was analyzed.

\section{The Circulatory system}

The CVS network is a directed graph (digraph). In this section, the matrices that describe the network of the CVS are constructed.

\subsection{Some elementary network lexica}

Let $(V, E)$ be a pair of non-void sets consisting of $V$, the set of vertices (nodes) on a network, and $E$, the set of edges (arcs) between pairs of the nodes on the network. The relation $G=(V, E)$ represents the graph of the network. A given finite directed graph (digraph), $G$, consists of a set of nodes, $V(G)$ such that,

$$
V(G)=v_{k} \quad k=1,2, \ldots, n
$$

alongside an edge set, $\varepsilon(G) \subseteq V(\mathrm{G}) \times V(\mathrm{G})$. If $v_{l}$ and $v_{m}$ are connected by an $\operatorname{arc}\left(v_{l}, v_{\mathrm{m}}\right)$, then $v_{l}$ and $v_{m}$ are end vertices of the edge $\left(v_{l}, v_{m}\right)$. The $\operatorname{arcs}\left(v_{l}, v_{m}\right)$ and $\left(v_{m}, v_{l}\right)$ are in opposite directions of a digraph. Since $\left(v_{l}, v_{\mathrm{m}}\right)$ is directed, $v_{m}$ is a direct successor of $v_{l}$ and $v_{l}$ is a direct predecessor of $v_{m}$. A network may consist of indegree $d^{-}\left(v_{l}\right)$ nodes and outdegree nodes $d^{+}\left(v_{l}\right)$. A source in a network is such that $d^{-}\left(v_{l}\right)=0$ and a sink is such that $d^{+}\left(v_{l}\right)=0$. A network may be a circuit, which is a closed path. A path is called a trial if any vertex is visited at most once except possibly the initial and terminal vertices when they are the same. 
In the diagrams below Figure 1 is the CVS network and Figure 2 is the prototype of Figure 1 used for the node-edge analysis of the CVS.

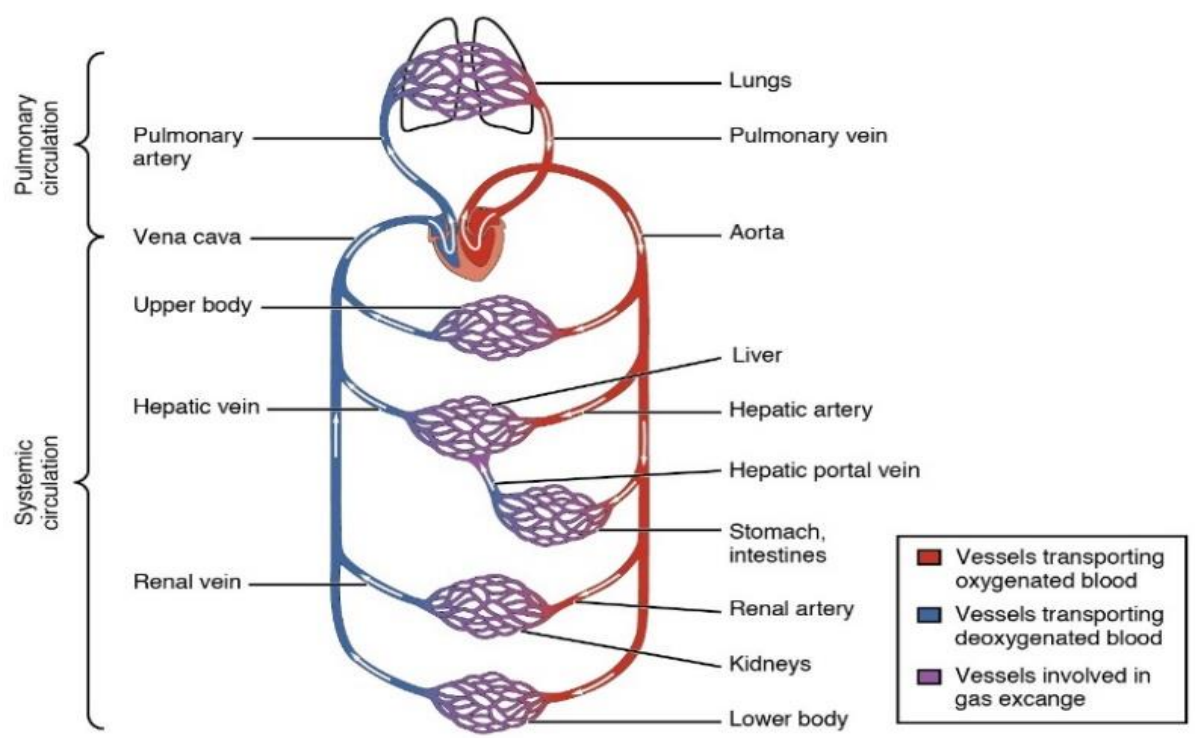

Figure 1: The cardiovascular system (CVS), "Blood Flow Through the Heart" By OpenStax College / CC BY 3.0 http://cnx.org/content/col

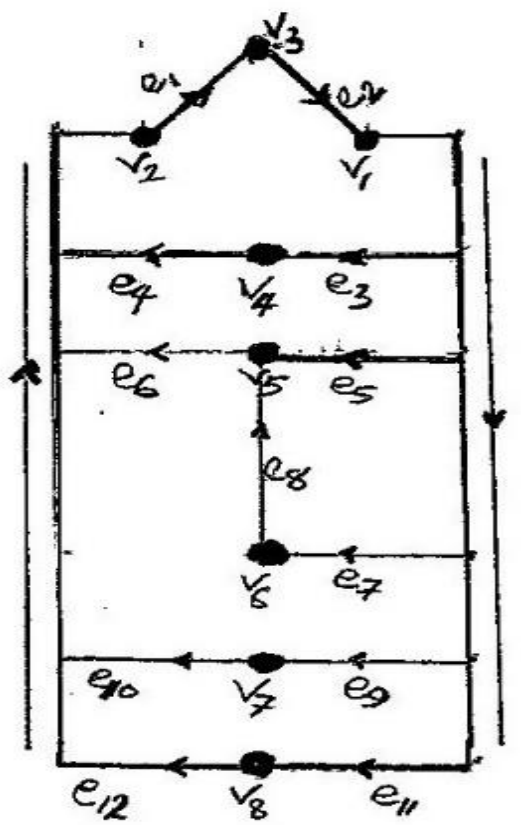

Figure 2: Node-edge schematic of the CVS 
In Figure 2 the arcs and the vertices are represented by $e_{i}(i=1,2, \ldots, 12)$ and $v_{i}(i$ $=1,2, \ldots, 8)$ respectively. The heart consists of two vertices denoted $v_{1}(\mathrm{LV})$ and $\mathrm{v}_{2}(\mathrm{RV})$; the lungs is denoted $v_{3}$; the upper body is denoted $v_{4}$; the liver is denoted $v_{5}$; the stomach/intestines is denoted $v_{6}$, kidneys denoted $v_{7}$ and lower body denoted v8. Since the CVS is a directed flow, arrowheads were used to indicate flow direction, as shown. The aorta (indicated by the downward pointing arrow) is the major arc through which subsidiary arcs connect node $v_{l}$ to their respective organs.

\subsection{The CVS matrix}

It is assumed that all edges connecting the nodes are rectifiable. Let $A_{i j}$ be the adjacency matrix of the given system. The required matrix satisfies the equation:

$A_{i j}=$ number of direct predecessors issuing from vertex $v_{i}$ into vertex $v_{j}$.

The adjacency matrix of the CVS is constructed below using Figure 2 and equation (2) above.

$$
A=\left(\begin{array}{lllllllll}
0 & 0 & 1 & 0 & 0 & 0 & 0 & 0 \\
0 & 0 & 0 & 1 & 1 & 0 & 1 & 1 \\
0 & 1 & 0 & 0 & 0 & 0 & 0 & 0 \\
1 & 0 & 0 & 0 & 0 & 0 & 0 & 0 \\
1 & 0 & 0 & 0 & 0 & 1 & 0 & 0 \\
1 & 0 & 0 & 0 & 0 & 0 & 0 & 0 \\
1 & 0 & 0 & 0 & 0 & 0 & 0 & 0 \\
1 & 0 & 0 & 0 & 0 & 0 & 0 & 0
\end{array}\right) \begin{aligned}
& v_{1} \\
& v_{2} \\
& v_{3} \\
& v_{4} \\
& v_{5} \\
& v_{6} \\
& v_{7} \\
& v_{8}
\end{aligned}
$$

In the above matrix, each $v_{i}$ on the row describes the initial vertex of an arc $e_{j}$ while each $v_{j}$ on the column describes the terminal vertex of the arc. Node $v_{1}$ represents the left side of the heart, which includes the left ventricle (LV), while $v_{2}$ represents the right side of the heart, which includes the right ventricle (RV). Observe that

$$
d^{-}\left(v_{1,2}\right)=1 \text { and } d^{+}\left(v_{1,2}\right)=1
$$

However, by the law of conservation, each of them serves as a source and a sink. 


\subsection{Construction of incidence matrix}

The flow arrangement of the CVS is developed using the incident matrix. Given an all-vertex incidence matrix, $B=\left(b_{i j}\right)$, then the matrix elements are determined by otherwise

$$
b_{i j}=\left\{\begin{array}{l}
1 \text { if } v_{i} \text { is the beggining vertex of } e_{j} \\
-1 \text { if } v_{i} \text { is the end vertex of } e_{j} \\
0 \quad \text { otherwise. }
\end{array}\right.
$$

From the foregoing one finds

$$
b_{i j}=\left(\begin{array}{cccccccccccc}
e_{1} & e_{2} & e_{3} & e_{4} & e_{5} & e_{6} & e_{7} & e_{8} & e_{9} & e_{10} & e_{11} & e_{12} \\
0 & -1 & 1 & 0 & 1 & 0 & 1 & 0 & 1 & 0 & 1 & 0 \\
1 & 0 & 0 & -1 & 0 & -1 & 0 & 0 & 0 & -1 & 0 & -1 \\
-1 & 1 & 0 & 0 & 0 & 0 & 0 & 0 & 0 & 0 & 0 & 0 \\
0 & 0 & -1 & 1 & 0 & 0 & 0 & 0 & 0 & 0 & 0 & 0 \\
0 & 0 & 0 & 0 & -1 & 1 & 0 & -1 & 0 & 0 & 0 & 0 \\
0 & 0 & 0 & 0 & 0 & 0 & -1 & 1 & 0 & 0 & 0 & 0 \\
0 & 0 & 0 & 0 & 0 & 0 & 0 & 0 & -1 & 1 & 0 & 0 \\
0 & 0 & 0 & 0 & 0 & 0 & 0 & 0 & 0 & 0 & -1 & 1
\end{array}\right) v_{2} v_{2}
$$

\subsection{Pulmonary and systemic circuit (cut) matrix}

Essentially the CVS is a closed system. An age-old study was conducted on open systems in biology by Von [16]. It was perceived that the LV circulation model assumes that the circulation is a closed system of vessels wherein the pressure gradient between the aorta and the right atrium regulates the flow and where, in a steady-state condition, the outputs of the left and right hearts are closely matched in keeping with the law of conservation of energy and matter Branko [17]. Circuit matrices could be produced each for the pulmonary flow from the heart-lungs and heart-main-body intermodal pathways, as shown in Figure $3 \mathrm{a}$ and Figure $3 \mathrm{~b}$ respectively below.

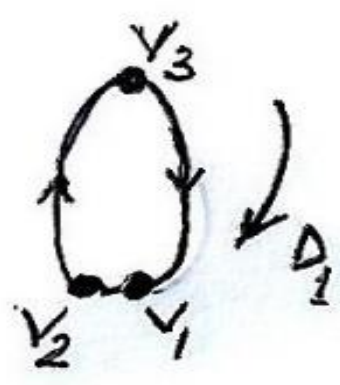

Figure 3a

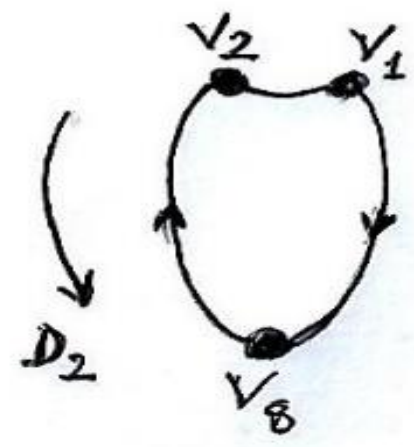

Figure 3b

Figure 3: Schematics for cut matrices of the CVS 
Give each of the circuits $D_{1}$ and $D_{2}$ an arbitrary orientation so as to define the circuit matrix. The circuit matrix is $D=\left(d_{i j}\right)$, where,

$$
d_{i j}= \begin{cases}1 & \text { whenever } \mathrm{e}_{\mathrm{j}} \text { is in } \mathrm{D}_{\mathrm{i}} \text { and they are in the same direction } \\ -1 & \text { whenever } \mathrm{e}_{\mathrm{j}} \text { is in } \mathrm{D}_{\mathrm{i}} \text { and they are in the reverse direction } \\ 0 & \text { else }\end{cases}
$$

The matrix is shown below

$$
\begin{aligned}
& \begin{array}{llll}
e_{1} & e_{2} & e_{3} & e_{4}
\end{array} \\
& d_{i j}=\left(\begin{array}{cccc}
1 & 1 & 0 & 0 \\
0 & 0 & -1 & -1
\end{array}\right) \begin{array}{l}
D_{1} \\
D_{2}
\end{array} \text {. }
\end{aligned}
$$

\subsection{Central point of CVS}

The CVS is the interplay of the heart and the vasculature to maintain the integrity of blood flow. The vascular structures are conduits through which blood and its contents are delivered to parts of the body, including the heart. The heart serves as a pump. This role makes it an active part of the system. The flow orientation of blood is controlled by the heart through special valves. At the entrance are the mitral valve (left) and the tricuspid valve (right), and at the exit of the ventricles are the aortic valve (left) and the pulmonic valve (right). In a proper working condition, the cardiac valves safeguard a one-way system of blood flow. Pressure situations in the ventricles as a result of the contraction of the heart muscles influence the valves. An increase in pressure has the effect of closing the inlet valve and opening the outlet valve. The pressure created by the left heart is approximately three times higher $(\approx 13 \mathrm{kPa})$ than that created by the right heart $(\approx 4 \mathrm{kPa})$, Nithiarasu [18]. This is so because the left ventricle requires much higher pressure to prosecute systemic circulation. Work done by pressure, $P$, on the surface area of the heart chamber is

$$
\Delta W=\int \frac{1}{A} F A d r=\int \frac{1}{A} F d V=\int_{V_{a}}^{V_{b}} P d V
$$

Thus, each heart compression induces the pumping of $V_{b}-V_{a}=\Delta V$ volume of blood. Typically, pressure rises from diastolic component $P_{d}$ gradually up to systolic component $P_{s}$ and falls back to diastolic pressure when the heart muscle relaxes. Now the work takes the form 


$$
\begin{aligned}
\Delta W & =\int_{V_{a}}^{V_{b}}\left(P_{d}+\frac{1}{\Delta V}\left(P s-P_{d}\right) \cdot\left(V-V_{1}\right)\right) d V \\
\cong & P_{d} \cdot \Delta V+\frac{1}{2}\left(P_{s}-P_{d}\right) \cdot \Delta V
\end{aligned}
$$

The mean ventricular blood pressure is $P_{d}+\frac{P_{s}-P_{d}}{2} \equiv \bar{P}$.

\section{Governing network equations}

In the network of elastic tubes physiological assumption of $1 \mathrm{D}$ blood flow model is the viscous incompressible fluid flow. In general, the hydrodynamic part of the model representing the fluid is obtained by the 3D Navier-Stokes equations and the elastic part of the model representing the cardio-vascular structures is described by deformable solid mechanics. The two parts are coupled, and therefore simulations of local flows in complicated regions (carotid arteries, large systemic arteries, etc.) is a coupled fluid-structure interaction (FSI) problem. Formaggia et al. [19], and Cani' $\mathrm{c}$ and Kim [20] derived 1D flow equations from averaging the 3D equations in a single vessel. In the hemodynamic model of global circulation 1D flow equations in distinct vessels with coupled boundary conditions at junction points between the vessels and at the heart are employed. A model of viscous incompressible fluid flow through an elastic tube is used in describing the $1 \mathrm{D}$ flow in a separate vessel.

Encode by $A(t, x)$ a vessel's cross-section area, by $u(t, x)$ the linear mean velocity over the cross-section. The governing equation of mass conservation is of the form,

$$
\frac{\partial A}{\partial t}+\frac{\partial(A u)}{\partial x}=h_{A}
$$

and the equation of momentum in the sense of Bernoulli is

$$
\frac{\partial A}{\partial t}+\frac{\partial\left(u^{2} / 2+p / \rho\right)}{\partial x}=\sum h_{u}
$$

In equation (11) $h_{A}$ encodes the mass source or sink situated at some point within a considered region. In the absence of a source, $h_{A}$ is zero. In the momentum equation (12), $p$ is the blood transmural pressure, $\rho$ is the blood density; the left-hand side terms embody inertia forces, while the right-hand side terms are an ensemble of forces which include traction and body forces and gravity, as the case may be. In this study, each of the organs is considered a blood source and/or sink with intensity. Thus, one may replace $h_{A}$ with $\delta_{A}$, where $\delta$ is the source or sink intensity. In accommodating gravitational impact on the systemic circulation, Buxton and Clarke [21] used source term in equation (12) in the form $h_{u}^{g}=g \sin \theta_{k}$ where $g$ is the gravity constant and $\theta$ is the angle between the vessel and the gravity field. Formaggia et al. [12] and Larrabidea et al. [22] supplied an alternative formulation 
of mass and momentum balance in $(A, Q, p)$ variables in which viscous flow resistance per unit length of the vessel was considered. Besides the compatibility conditions required along the characteristics of equations (11)-(12), the 1D blood flow models are required at junction consisting of $N$ vessel must admit mass conservation on the vessel network. It is of note that the boundary conditions have to take account of compatibility conditions along characteristics of equations (11)(12). It was observed (Sherwin et al. [23], van de Vosse and Stergiopulos [24], Bessonov et al. [25] that there exists an incoming and outgoing characteristic for every vessel endpoint. At the junction of $N$ vessels, the conventional boundary condition caters for mass conservation

Suppose a junction contains $N$ vessels. The boundary condition admits the mass conservation

$$
\sum_{r=r_{1}, r_{2}, \ldots, r_{N}} \lambda_{r} A_{r} u_{r}=0
$$

where $A_{r}=A_{r}\left(t, \tilde{x}_{r}\right), \quad u_{r}=u_{r}\left(t, \tilde{x}_{r}\right)$, and $\left\{r_{1}, \ldots, r_{N}\right\}$ enlist the incident vessels;

$$
\lambda_{r}= \begin{cases}1 & \text { for inward bound vessels, } \\ -1 & \text { for outward bound vessels, }\end{cases}
$$

and

$$
\tilde{x}_{r}= \begin{cases}0 & \text { for inward bound vessels } \\ J_{r} & \text { for outward bound vessels. }\end{cases}
$$

The total pressure continuity conditions using Bernoulli integral conservation is such that

$$
\frac{u_{r}^{2}}{2}+\frac{p_{r}\left(A_{r}\right)}{\rho}=P^{k}
$$

where $P^{k}$ encodes the aggregate pressure at the junction with index $k$.

Now we give the network expression for blood mass and flow. Every edge in the CVS describes a blood vessel. In the network structure it is assumed that the organs, $v_{\mathrm{i}}$ earlier specified are sources and sinks. The edges (arcs) $e_{\mathrm{i}}$ enhance the delivery of the essential mass (blood with nutrients). A general mass $(M)$ - flow $(f)$ linear relationship reads,

$$
M_{v_{j} v_{k}}^{(x y)}=R_{v_{j} v_{k}} f_{v_{j} v_{k}}^{(x y)}
$$

In equation (17) the left-hand side encodes the mass being transported across the edge joining nodes $v_{\mathrm{j}}$ and $v_{k}$ of the network with a source located at node $x$ and a sink located at node $y$. With many sources and sinks, more upper indexes may be added to the equation. The right-hand side of equation (17) is the product of the 
resistance $R_{v_{j} v_{k}}$ of the edge and the mass flow $f_{v_{j} v_{k}}^{(x y)}$. About the present consideration take, for instance, the flow of blood from node $v_{l}$ (the left ventricle, to $v_{4}$ (the upper body) [note that the left ventricle is the pump for the systemic circuit, which delivers the blood to the tissue cells of the body]. The quantity (mass) of blood $M$ is transported through the aorta to $e_{3}$ (aortic branch). Within this tract, $v_{1}$ is the source of supply to $v_{4}$ (the sink), and from outside the track, $v_{3}$ (the lungs) is the source of supply to $v_{l}$. Thus, along the given trajectory, equation (16) takes the form

$$
M_{\underline{v_{1} v_{4}}}^{\left(v_{1} v_{4}\right)}=R_{\underline{v_{1} v_{4}}} f_{\underline{v_{1} v_{4}}}^{\left(v_{1} v_{4}\right)}
$$

where the under arrows indicate the edge connecting the nodes $v_{l}$ and $v_{4}$. When conservative flow is assumed, as it is essential here, the net flow at any node $v_{i}$ other than the source and the sink nodes in the network is zero (i.e. $\left(\sum_{k=1}^{N} f_{v_{j} v_{k}}^{(x)}=0\right)$ ). As the source and sink are likened to a dipole, the net flows are represented by $I$ and $-I$ respectively. Now the net flow at node $v_{j}$ is

$$
\sum_{k=1}^{N} f_{v_{j} v_{k}}^{(x y)}=I\left(\delta_{v_{j} x}-\delta_{v_{k} y}\right)=\sum_{k=1}^{N} \Omega_{j k}\left(V_{j}^{(x y)}-V_{k}^{(x y)}\right)
$$

where $V_{j}^{(x y)}$ is the potential at node $v_{j}$ to some $x$-y pair, $\Omega_{j k}=A_{j k} / R_{j k}$ encodes the matrix representation of the network structure. Here $A_{j k}$ is the adjacency matrix entries (see equation (3)).

It is of note that every node has an accompanying blood pressure value and the pressure difference between abutting nodes drive the flow through the connecting vessels. The conductance $G_{e i}$ of such a vessel reads:

$$
G_{e_{i}}=\lambda \frac{\rho A^{2}}{8 \pi \mu L}
$$

where, $L$ is the length of the vessel, $\mu$ and $\rho$ are the dynamic viscosity and blood density respectively. For a Hagen-Poiseuille flow through a perfect cylindrical vessel $\lambda=1$ in equation (20), which however amounts to a geometrical idealization. The effective conductance between two adjacent nodes connected by more than one edge is the sum of the individual vessel conductance. The mass flow rate $M$ through a vessel is the product of its conductance and the pressure difference $\Delta p$ across its length. Thus,

$$
M=G_{e_{i}} \Delta p=\lambda \frac{\rho A^{2}}{8 \pi \mu L} \Delta p
$$




\subsection{CVS resistance}

The right-hand side of equation (17) comprises the resistance to flow $R$, a proportionality factor that depends on the structure of the edge and the unidentified flow $f$ which the edge accommodates for a given site of the supply-demand nodes. This resistance to flow $f$ may be determined by

$$
R=\frac{\Delta P}{f}\left[\frac{m m H g}{l / s}\right]
$$

Determination of $R$ presupposes the knowledge of $f$. The CVS is stocked with mini aortic bifurcations (see Figure 4) by which daughter arcs (vessels) supply blood to the organs.

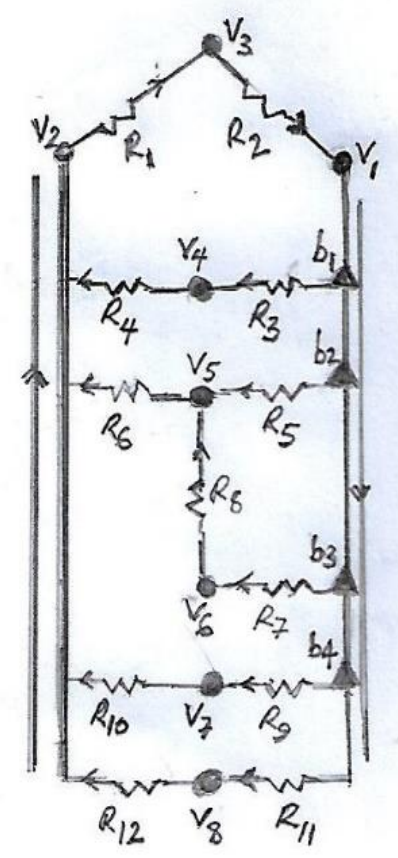

Figure 4: CVS resistive network showing resistors $R_{\mathrm{i}}$ and bifurcation points $b_{\mathrm{i}}$

With brief attention to arrangement, there are both series and parallel settings. Resistances in each case are governed by elementary circuit law, whereby for two given resistors $R_{\mathrm{a}}$ and $R_{\mathrm{b}}$, say, the series arrangement yields

$$
R=R_{a}+R_{b}
$$

and the parallel arrangement of the vessels such that

$$
\frac{1}{R}=\frac{1}{R_{a}}+\frac{1}{R_{b}} \text { or } R=\frac{R_{a} \cdot R_{b}}{R_{a}+R_{b}}
$$


The series arrangement is seen to hold from arterial compartments to the venous compartments, with minor exceptions. At the venous compartment, the daughter vessels are tributaries that return oxygen-depleted blood to the right atrium of the heart. Whichever vessel arrangement applies to any section of the system, the edge capacity $C_{v_{j} v_{k}}^{(x y)}$ which any supply-demand network must attain to escape over-loads is such that

$$
\left|M_{v_{j} v_{k}}^{(x y)}\right| \leq C_{v_{j} v_{k}}^{(x y)}
$$

In a physiological state, the edge capacity $C_{v_{j} v_{k}}^{(x y)}$ is independent of the demandsupply site within the network (in effect, $C_{v_{j} v_{k}}^{(x y)}=C_{v_{j} v_{k}}$ ). It is a function of the equivalent resistance of the edge and it is proportional to the total mass of blood that is pumped by the right ventricle per unit of time. (A good work on pressureflow models in pulmonary and systemic circulation may be found in Ketan and Bhathawala [7]).

\subsection{Wave propagation at a bifurcation}

Many (daughter vessels) branch from the aorta to the organs of the body (see Figure 4). Although the aortic bifurcation is the point at which the abdominal aorta bifurcates into the left and right common iliac arteries, we consider each of the branch points of the daughter- vessels as a mini bifurcation point. At points of bifurcation, the pressure wave is to some extent transmitted downstream into the daughter-vessels and eventually partially reflected backward. Consequently, bifurcation induces incident wave $I$, moving downward, the reflected wave (ref.) propagating upward together with two transmitted waves $\left(w_{1}\right.$ and $\left.w_{2}\right)$ downward. As earlier stated, the daughter vessels return oxygen-depleted blood to the right atrium of the heart at venous compartment.

If pressure is assumed to take the same values independent of the vessels, then the continuity of pressure may be expressed at the junction in the form

$$
\sum p_{W}=p_{w_{1}}=p_{w_{2}}
$$

where $P_{W}$ encodes the incident and the reflected waves. The conservation of mass that relates to discharges is in the form,

$$
M_{I}-M_{r e f .}=\sum_{i=1}^{2} M_{w_{i}}
$$


When the pressure-discharge relationship is put in the form

$$
M=\frac{A}{\rho c} p
$$

and relating the discharge $M$ to the vessel's characteristics, $Z$ (known as the impedance) one finds that

$$
M=\frac{p}{Z}, \quad Z=\frac{\rho c}{A} .
$$

Substituting equation (28a) in equation (26), this and equation (25) give the system

$$
\left.\begin{array}{l}
\sum p_{W}=p_{w_{1}} \\
\sum p_{W}=p_{w_{2}} \\
\frac{\sum p_{W}}{Z_{0}}=\frac{p_{w_{1}}}{Z_{1}}+\frac{p_{w_{2}}}{Z_{2}}
\end{array}\right\}
$$

where the subscript 0 represents the parent vessel and the quantities 1 and 2 represent the two daughters. From the foregoing, the incident and reflected waves relate by

$$
\frac{p_{I}-p_{\text {ref. }}}{Z_{0}}=\frac{p_{I}+p_{\text {ref. }} .}{Z_{1}}+\frac{p_{I}+p_{\text {ref. }} .}{Z_{2}} .
$$

The ratio $p_{r} / p_{i}=R_{Z}$ is the reflection coefficient given by

$$
R_{Z}=Z^{\alpha} / Z^{\beta}
$$

where

$$
Z^{\alpha}=\frac{1}{Z_{0}}-\frac{1}{Z_{1}}-\frac{1}{Z_{2}} \text { and } Z^{\beta}=\frac{1}{Z_{0}}+\frac{1}{Z_{1}}+\frac{1}{Z_{2}}
$$

The aortic bifurcation into the left and right common iliac arteries is an exemplary site for effectively reflected waves. Since real bifurcation furnishes a non-zero small reflection, each of the mini bifurcation points considered here is expected to induce a negligible reflection under physiological pressure conditions.

\section{Electrical analogy}

First, consider the network as analogous to an electric circuit. The CVS network may be characterized by a set of network constraints. They are the branch (edge) or element-based constraints, and the non-element-based topological constraints, emanating from Kirchhoff's Current Law (KCL) and Voltage Law (KVL). In the circulatory network, each of the edges is an analog of an electric wire with some specific resistance. Thus, Ohm's law applies to the network. Accordingly, the voltage drop $V$ between the ends of each wire obeys the equation $V=I R$, where $I$ is the current in the wire and $R$ its resistance. In a node where branch currents $x_{1}, \ldots, x_{n}$ 
enter, Kirchhoff's current law (KCL) states that the sum of all branch current entering a node equals zero. Accordingly,

$$
\sum_{i=1}^{n} \mathbf{x}_{i}=0
$$

Correspondingly, by Kirchhoff's voltage law (KVL),

$$
\sum_{i=1}^{n} u_{i}=0
$$

where $u_{i}$ encodes voltage drop in the circuit. Equations for the network shall be derived, and KCL and KVL are to be applied together with Ohm's law.

\subsection{Arcs and resistances}

Suppose there is a voltage source $r_{0}$, together with the resistances $R_{1}, \ldots, R_{12}$. The measurement of voltage drop, $u_{i}$, through the resistances is taken concerning the equivalent arcs, $e_{i}$. From the topology under consideration one finds the following:

$$
\begin{array}{lll}
u_{1}=v_{2}-v_{3} & u_{5}=v_{1}-v_{5} & u_{9}=v_{1}-v_{7} \\
u_{2}=v_{3}-v_{1} & u_{6}=v_{5}-v_{2} & u_{10}=v_{7}-v_{2} \\
u_{3}=v_{1}-v_{4} & u_{7}=v_{1}-v_{6} & u_{11}=v_{1}-v_{8} \\
u_{4}=v_{4}-v_{2} & u_{8}=v_{6}-v_{5} & u_{12}=v_{8}-v_{2}
\end{array}
$$

The vector arrangement yields,

$$
\left[\begin{array}{l}
u_{1} \\
u_{2} \\
u_{3} \\
u_{4} \\
u_{5} \\
u_{6} \\
u_{7} \\
u_{8} \\
u_{9} \\
u_{10} \\
u_{11} \\
u_{12}
\end{array}\right]=\left[\begin{array}{cccccccc}
0 & 1 & -1 & 0 & 0 & 0 & 0 & 0 \\
-1 & 0 & 1 & 0 & 0 & 0 & 0 & 0 \\
1 & 0 & 0 & -1 & 0 & 0 & 0 & 0 \\
0 & -1 & 0 & 1 & 0 & 0 & 0 & 0 \\
1 & 0 & 0 & 0 & -1 & 0 & 0 & 0 \\
0 & -1 & 0 & 0 & 1 & 0 & 0 & 0 \\
1 & 0 & 0 & 0 & 0 & -1 & 0 & 0 \\
0 & 0 & 0 & 0 & -1 & 1 & 0 & 0 \\
1 & 0 & 0 & 0 & 0 & 0 & -1 & 0 \\
0 & -1 & 0 & 0 & 0 & 0 & 1 & 0 \\
1 & 0 & 0 & 0 & 0 & 0 & 0 & -1 \\
0 & -1 & 0 & 0 & 0 & 0 & 0 & 1
\end{array}\right]\left[\begin{array}{c}
v_{1} \\
v_{2} \\
v_{3} \\
v_{4} \\
v_{5} \\
v_{6} \\
v_{7} \\
v_{8}
\end{array}\right]
$$


Ground one node $v_{2}$, say. That is, take $v_{2}$ as the reference node. (Note that the choice is arbitrary). From equation (33) above one finds that

$$
\left[\begin{array}{l}
u_{1} \\
u_{2} \\
u_{3} \\
u_{4} \\
u_{5} \\
u_{6} \\
u_{7} \\
u_{8} \\
u_{9} \\
u_{10} \\
u_{11} \\
u_{12}
\end{array}\right]=\left[\begin{array}{ccccccc}
0 & -1 & 0 & 0 & 0 & 0 & 0 \\
-1 & 1 & 0 & 0 & 0 & 0 & 0 \\
1 & 0 & -1 & 0 & 0 & 0 & 0 \\
0 & 0 & 1 & 0 & 0 & 0 & 0 \\
1 & 0 & 0 & -1 & 0 & 0 & 0 \\
0 & 0 & 0 & 1 & 0 & 0 & 0 \\
1 & 0 & 0 & 0 & -1 & 0 & 0 \\
0 & 0 & 0 & -1 & 1 & 0 & 0 \\
1 & 0 & 0 & 0 & 0 & -1 & 0 \\
0 & 0 & 0 & 0 & 0 & 1 & 0 \\
1 & 0 & 0 & 0 & 0 & 0 & -1 \\
0 & 0 & 0 & 0 & 0 & 0 & 1
\end{array}\right]\left[\begin{array}{c}
v_{1} \\
v_{3} \\
v_{4} \\
v_{5} \\
v_{6} \\
v_{7} \\
v_{8}
\end{array}\right]
$$

In vector form equation (34) gives

$$
\mathbf{u}=\mathbf{C v}
$$

where the matrix $C$ denotes network connectivity. Ohm's Law, " $I=V / R$ ", connects the voltage drop across each resistor to the current. In line with the resistors, Ohm's Law reads,

$$
x_{j}=\frac{u_{j}}{R_{j}} \quad j=1,2, \ldots, 8
$$

The matrix-vector arrangement reads:

$$
\left[\begin{array}{l}
x_{1} \\
x_{2} \\
x_{3} \\
x_{4} \\
x_{5} \\
x_{6} \\
x_{7} \\
x_{8} \\
x_{9} \\
x_{10} \\
x_{11} \\
x_{12}
\end{array}\right]=\left[\begin{array}{cccccccccccc}
1 / R_{1} & 0 & 0 & 0 & 0 & 0 & 0 & 0 & 0 & 0 & 0 & 0 \\
0 & 1 / R_{2} & 0 & 0 & 0 & 0 & 0 & 0 & 0 & 0 & 0 & 0 \\
0 & 0 & 1 / R_{3} & 0 & 0 & 0 & 0 & 0 & 0 & 0 & 0 & 0 \\
0 & 0 & 0 & 1 / R_{4} & 0 & 0 & 0 & 0 & 0 & 0 & 0 & 0 \\
0 & 0 & 0 & 0 & 1 / R_{5} & 0 & 0 & 0 & 0 & 0 & 0 & 0 \\
0 & 0 & 0 & 0 & 0 & 1 / R_{6} & 0 & 0 & 0 & 0 & 0 & 0 \\
0 & 0 & 0 & 0 & 0 & 0 & 1 / R_{7} & 0 & 0 & 0 & 0 & 0 \\
0 & 0 & 0 & 0 & 0 & 0 & 0 & 1 / R_{8} & 0 & 0 & 0 & 0 \\
0 & 0 & 0 & 0 & 0 & 0 & 0 & 0 & 1 / R_{9} & 0 & 0 & 0 \\
0 & 0 & 0 & 0 & 0 & 0 & 0 & 0 & 0 & 1 / R_{10} & 0 & 0 \\
0 & 0 & 0 & 0 & 0 & 0 & 0 & 0 & 0 & 0 & 1 / R_{11} & 0 \\
0 & 0 & 0 & 0 & 0 & 0 & 0 & 0 & 0 & 0 & 0 & 1 / R_{12}
\end{array}\right]\left[\begin{array}{l}
u_{1} \\
u_{2} \\
u_{3} \\
u_{4} \\
u_{5} \\
u_{6} \\
u_{7} \\
u_{8} \\
u_{9} \\
u_{10} \\
u_{11} \\
u_{12}
\end{array}\right]
$$


The above matrix equation is of the form,

$$
\mathbf{x}=\mathbf{M u}
$$

where $\mathbf{M}$ denotes the dynamics of the network. The KCL admits the product:

$\left[\begin{array}{cccccccccccc}0 & -1 & 1 & 0 & 1 & 0 & 1 & 0 & 1 & 0 & 1 & 0 \\ -1 & 1 & 0 & 0 & 0 & 0 & 0 & 0 & 0 & 0 & 0 & 0 \\ 0 & 0 & -1 & 1 & 0 & 0 & 0 & 0 & 0 & 0 & 0 & 0 \\ 0 & 0 & 0 & 0 & -1 & 1 & 0 & -1 & 0 & 0 & 0 & 0 \\ 0 & 0 & 0 & 0 & 0 & 0 & -1 & 1 & 0 & 0 & 0 & 0 \\ 0 & 0 & 0 & 0 & 0 & 0 & 0 & 0 & -1 & 1 & 0 & 0 \\ 0 & 0 & 0 & 0 & 0 & 0 & 0 & 0 & 0 & 0 & -1 & 1\end{array}\right]\left[\begin{array}{c}x_{1} \\ x_{2} \\ x_{3} \\ x_{4} \\ x_{5} \\ x_{6} \\ x_{7} \\ x_{8} \\ x_{9} \\ x_{10} \\ x_{11} \\ x_{12}\end{array}\right]=\left[\begin{array}{l}0 \\ 0 \\ 0 \\ 0 \\ 0 \\ 0 \\ 0\end{array}\right]$

The equation (39) is of the form

$$
\mathbf{C}^{\mathbf{T}} \mathbf{x}=\mathbf{0}
$$

Introduce ( $\mathbf{x}=\mathbf{M u})$ for $\mathbf{x}$ into equation (40) to get

$$
\mathbf{C}^{\mathrm{T}} \mathbf{M u}=\mathbf{0}
$$

The network's equilibrium condition must be attained at a constant voltage source, $r_{0}$. Therefore,

Then

$$
\mathbf{u}=\mathbf{r}-\mathbf{C v}
$$

$$
\mathbf{C}^{\mathrm{T}} \mathbf{M u}=\mathbf{C}^{\mathrm{T}} \mathbf{M}(\mathbf{r}-\mathbf{C v})
$$

Thus,

$$
\mathbf{C}^{\mathrm{T}} \mathrm{KCv}=\mathbf{H}
$$

where $\mathbf{H}=\mathbf{C}^{\mathrm{T}} \mathbf{M r}$. $\mathbf{H}$ encodes the vector of current sources which represents the network's stimuli. 


\subsection{Characteristic equations for vi}

The individual node potential of the system may be calculated. First, compute $\mathbf{C}^{\mathrm{T}} \mathbf{M}$ :

$$
\boldsymbol{C}^{T} \boldsymbol{M}=\left[\begin{array}{cccccccccccc}
0 & -1 & 1 & 0 & 1 & 0 & 1 & 0 & 1 & 0 & 1 & 0 \\
-1 & 1 & 0 & 0 & 0 & 0 & 0 & 0 & 0 & 0 & 0 & 0 \\
0 & 0 & -1 & 1 & 0 & 0 & 0 & 0 & 0 & 0 & 0 & 0 \\
0 & 0 & 0 & 0 & -1 & 1 & 0 & -1 & 0 & 0 & 0 & 0 \\
0 & 0 & 0 & 0 & 0 & 0 & -1 & 1 & 0 & 0 & 0 & 0 \\
0 & 0 & 0 & 0 & 0 & 0 & 0 & 0 & -1 & 1 & 0 & 0 \\
0 & 0 & 0 & 0 & 0 & 0 & 0 & 0 & 0 & 0 & -1 & 1
\end{array}\right]
$$$$
\left[\begin{array}{cccccccccccc}
1 / R_{1} & 0 & 0 & 0 & 0 & 0 & 0 & 0 & 0 & 0 & 0 & 0 \\
0 & 1 / R_{2} & 0 & 0 & 0 & 0 & 0 & 0 & 0 & 0 & 0 & 0 \\
0 & 0 & 1 / R_{3} & 0 & 0 & 0 & 0 & 0 & 0 & 0 & 0 & 0 \\
0 & 0 & 0 & 1 / R_{4} & 0 & 0 & 0 & 0 & 0 & 0 & 0 & 0 \\
0 & 0 & 0 & 0 & 1 / R_{5} & 0 & 0 & 0 & 0 & 0 & 0 & 0 \\
0 & 0 & 0 & 0 & 0 & 1 / R_{6} & 0 & 0 & 0 & 0 & 0 & 0 \\
0 & 0 & 0 & 0 & 0 & 0 & 1 / R_{7} & 0 & 0 & 0 & 0 & 0 \\
0 & 0 & 0 & 0 & 0 & 0 & 0 & 1 / R_{8} & 0 & 0 & 0 & 0 \\
0 & 0 & 0 & 0 & 0 & 0 & 0 & 0 & 1 / R_{9} & 0 & 0 & 0 \\
0 & 0 & 0 & 0 & 0 & 0 & 0 & 0 & 0 & 1 / R_{10} & 0 & 0 \\
0 & 0 & 0 & 0 & 0 & 0 & 0 & 0 & 0 & 0 & 1 / R_{11} & 0 \\
0 & 0 & 0 & 0 & 0 & 0 & 0 & 0 & 0 & 0 & 0 & 1 / R_{12}
\end{array}\right]
$$

$$
=\left[\begin{array}{cccccccccccc}
0 & -1 / R_{2} & 1 / R_{3} & 0 & 1 / R_{5} & 0 & 1 / R_{7} & 0 & 1 / R_{9} & 0 & 1 / R_{11} & 0 \\
-1 / R_{1} & 1 / R_{2} & 0 & 0 & 0 & 0 & 0 & 0 & 0 & 0 & 0 & 0 \\
0 & 0 & -1 / R_{3} & 1 / R_{4} & 0 & 0 & 0 & 0 & 0 & 0 & 0 & 0 \\
0 & 0 & 0 & 0 & -1 / R_{5} & 1 / R_{6} & 0 & -1 / R_{8} & 0 & 0 & 0 & 0 \\
0 & 0 & 0 & 0 & 0 & 0 & -1 / R_{7} & 1 / R_{8} & 0 & 0 & 0 & 0 \\
0 & 0 & 0 & 0 & 0 & 0 & 0 & 0 & -1 / R_{9} & 1 / R_{10} & 0 & 0 \\
0 & 0 & 0 & 0 & 0 & 0 & 0 & 0 & 0 & 0 & -1 / R_{11} & 1 / R_{12}
\end{array}\right]
$$


Compute the matrix $\left(\mathbf{C}^{\mathbf{T}} \mathbf{M}\right) \mathbf{C}$ :

$\left(\mathbf{C}^{\mathrm{T}} \mathbf{M}\right) \mathbf{C}=\left[\begin{array}{cccccccccccc}0 & -1 / R_{2} & 1 / R_{3} & 0 & 1 / R_{5} & 0 & 1 / R_{7} & 0 & 1 / R_{9} & 0 & 1 / R_{11} & 0 \\ -1 / R_{1} & 1 / R_{2} & 0 & 0 & 0 & 0 & 0 & 0 & 0 & 0 & 0 & 0 \\ 0 & 0 & -1 / R_{3} & 1 / R_{4} & 0 & 0 & 0 & 0 & 0 & 0 & 0 & 0 \\ 0 & 0 & 0 & 0 & -1 / R_{5} & 1 / R_{6} & 0 & -1 / R_{8} & 0 & 0 & 0 & 0 \\ 0 & 0 & 0 & 0 & 0 & 0 & -1 / R_{7} & 1 / R_{8} & 0 & 0 & 0 & 0 \\ 0 & 0 & 0 & 0 & 0 & 0 & 0 & 0 & -1 / R_{9} & 1 / R_{10} & 0 & 0 \\ 0 & 0 & 0 & 0 & 0 & 0 & 0 & 0 & 0 & 0 & -1 / R_{11} & 1 / R_{12}\end{array}\right]\left[\begin{array}{ccccccc}0 & -1 & 0 & 0 & 0 & 0 & 0 \\ -1 & 1 & 0 & 0 & 0 & 0 & 0 \\ 1 & 0 & -1 & 0 & 0 & 0 & 0 \\ 0 & 0 & 1 & 0 & 0 & 0 & 0 \\ 1 & 0 & 0 & -1 & 0 & 0 & 0 \\ 0 & 0 & 0 & 1 & 0 & 0 & 0 \\ 1 & 0 & 0 & 0 & -1 & 0 & 0 \\ 0 & 0 & 0 & -1 & 1 & 0 & 0 \\ 1 & 0 & 0 & 0 & 0 & -1 & 0 \\ 0 & 0 & 0 & 0 & 0 & 1 & 0 \\ 1 & 0 & 0 & 0 & 0 & 0 & -1 \\ 0 & 0 & 0 & 0 & 0 & 0 & 1\end{array}\right]$

$$
=\left[\begin{array}{ccccccc}
\frac{1}{R_{2}}+\sum_{j=1}^{5} \frac{1}{R_{(2 j+1)}} & -1 / R_{2} & -1 / R_{3} & -1 / R_{5} & -1 / R_{7} & -1 / R_{9} & -1 / R_{11} \\
1 / R_{2} & 1 / R_{1}+1 / R_{2} & 0 & 0 & 0 & 0 & 0 \\
-1 / R_{3} & 0 & 1 / R_{3}+1 / R_{4} & 0 & 0 & 0 & 0 \\
-1 / R_{5} & 0 & 0 & 1 / R_{5}+1 / R_{6}+1 / R_{8} & -1 / R_{8} & 0 & 0 \\
-1 / R_{7} & 0 & 0 & -1 / R_{8} & 0 & 0 & 0 \\
-1 / R_{9} & 0 & 0 & 0 & 0 & 1 / R_{9}+1 / R_{10} & 0 \\
-1 / R_{11} & 0 & 0 & 0 & 0 & 0 & 1 / R_{11}+1 / R_{12}
\end{array}\right]
$$

When the inverse of the symmetric matrix $\left(\mathbf{C}^{\mathbf{T}} \mathbf{M}\right) \mathbf{C}$ is applied to $\mathbf{H}$, the vector of potentials $v_{i}$ are obtained, assuming $R_{i}$ are known.

Next, evaluate the vector $\mathbf{H}$,

$$
\mathbf{H}=\left(\mathbf{C}^{\mathrm{T}} \mathbf{M}\right) \mathbf{r}=\left[\begin{array}{cccccccccccc}
0 & -1 / R_{2} & 1 / R_{3} & 0 & 1 / R_{5} & 0 & 1 / R_{7} & 0 & 1 / R_{9} & 0 & 1 / R_{11} & 0 \\
-1 / R_{1} & 1 / R_{2} & 0 & 0 & 0 & 0 & 0 & 0 & 0 & 0 & 0 & 0 \\
0 & 0 & -1 / R_{3} & 1 / R_{4} & 0 & 0 & 0 & 0 & 0 & 0 & 0 & 0 \\
0 & 0 & 0 & 0 & -1 / R_{5} & 1 / R_{6} & 0 & -1 / R_{8} & 0 & 0 & 0 & 0 \\
0 & 0 & 0 & 0 & 0 & 0 & -1 / R_{7} & 1 / R_{8} & 0 & 0 & 0 & 0 \\
0 & 0 & 0 & 0 & 0 & 0 & 0 & 0 & -1 / R_{9} & 1 / R_{10} & 0 & 0 \\
0 & 0 & 0 & 0 & 0 & 0 & 0 & 0 & 0 & 0 & -1 / R_{11} & 1 / R_{12}
\end{array}\right]\left[\begin{array}{c}
r_{0} \\
0 \\
0 \\
0 \\
0 \\
0 \\
0 \\
0 \\
0 \\
0 \\
0 \\
0 \\
0 \\
0 \\
0 \\
0 \\
0 \\
0 \\
0 \\
0
\end{array}\right]=\left[\begin{array}{c}
0 \\
0 \\
0
\end{array}\right]
$$


Finally,

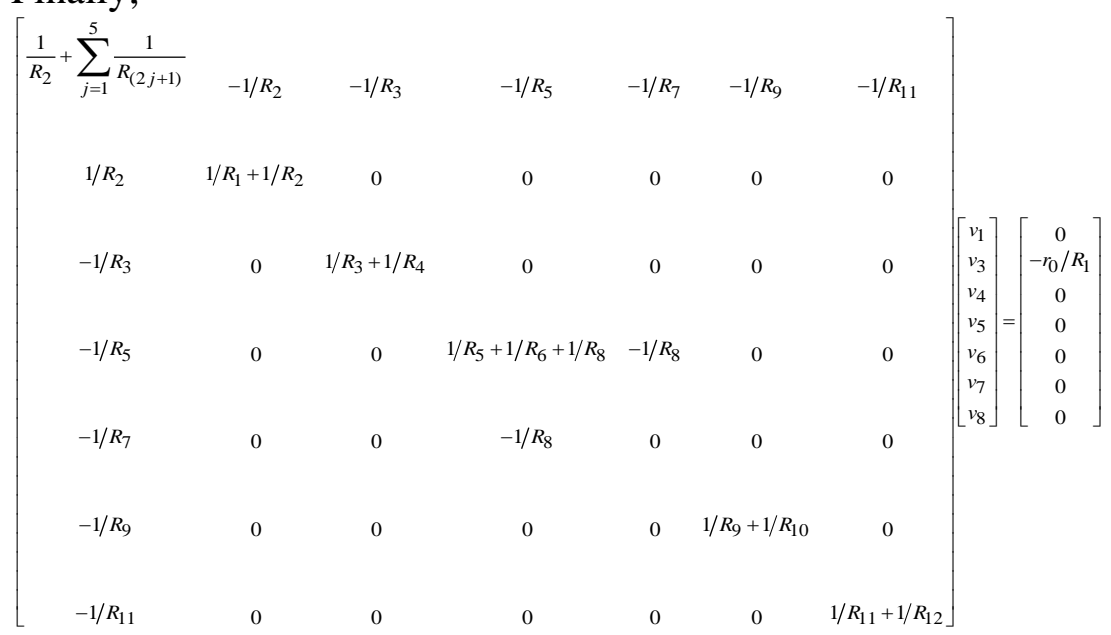

The values of $v_{1}, v_{3}, \ldots, v_{6}$ may be easily determined if the values of $R_{1}, R_{2}, \ldots, R_{7}$ are known.

\section{Conclusion}

The CVS, which supplies the human organs with blood, comprises the heart and a network of tubes. It has two modes of action: pulmonary circulation and, systemic circulation. Pulmonary circulation, known as the small circulation, is evident only between the heart and the lungs. The large (systemic) circulation takes oxygenated blood from the left ventricle through the aorta to the different organs through the arterial system.

In this work, some elements of graph theory together with electrical analogy were used in analyzing CVS network. Electrical flow is a directed flow. Therefore the representative graph is a directed graph (digragh). Away from here, the cardiac conduction system describing the cardiac electrical system was treated; the conduction mechanism drives blood flow. The parameters of great interest in this study are those of the nodes and the arcs if valvular and ventricular normality is presupposed. Some relevant questions are: (i) What are the likely causes of insufficient supplies to an organ? (ii) What may cause general poor circulation? (iii) Are such problems tractable? Recourse to the mass-flow linear relationship may provide some clue to the posers above. The mass flow rate, $M$, through a vessel, is the product of its conductance and the pressure difference $\Delta p$ across its length. The flow rate may be niggardly at a relatively low physiological pressure difference. The resistivity of each arc affects the flow rate. It is pertinent to determine the resistivity of each arc of the network in a bid to stage their possible pathogenesis and, by extension, those of their dependent organs.

This work did not detail microcirculation in which arterioles, venules, and capillaries play a dominant role. However, it is a prototype of the general case treated here in which the major organs were exemplified. 


\section{References}

[1] Jarvis, S. (2018). Vascular system 1: anatomy and physiology.Nursing Times, pp.114:4,40-44.https://www.nursingtimes.net/clinical-archive/cardiovascularclinical-archive/vascular-system-1-anatomy-and-physiology-26-03-2018/.

[2] Matthews, B. E., Oliver, M.F. and Rogers, M. E. "Circulatory system". Encyclopedia Britannica, https://www.britannica.com/science/circulatorysystem.

[3] Jarvis, S. and Saman, S. (2018). Cardiac system 1: anatomy and physiology. Nursing Times 2018, 114: 2, 34-37. https://www.nursingtimes.net/clinicalarchive/cardiovascular-clinical-archive/cardiac-system-1-anatomy-andphysiology-29-01-2018/.

[4] Smith, N.P., Pullan, A. J. and Hunter, P.J. (2002). An anatomically based model of transient coronary blood flow in the heart, SIAM J. Appl. Math., Vol. 62, 2002, pp. 990-1018.

[5] Ruan, W., Clark, M.E., Zhao, M. and Curcio, A. A hyperbolic system in a onedimensional network, arXiv: math-ph/0209015.

[6] Johannes, R., Marco, S. A., Lena, K., Alfred, B., Patrick, J. and Bruno, W. (2009). Vascular graph model to simulate the cerebral blood flow in realistic vascular networks, Journal of Cerebral Blood Flow \& Metabolism Vol. 29, 2009,pp 1429-1443.

[7] Ketan, N. and Bhathawala, P. H. (2017). Mathematical Modeling of Human Cardiovascular System: A Lumped Parameter Approach and Simulation, World Academy of Science, Engineering and Technology International Journal of Mathematical and Computational Sciences Vol:11, No:2. 2017.

[8] Rideout, V.C. and Katra, J.A. (1969). Computer Study of the Pulmonary Circulation, Simulation, Vol. 12, No. 5, 1969, pp.239-245.

[9] Rideout, V.C. and Dick, D.E. (1967). Difference-Differential Equations for Fluid Flow in Distensible Tubes, IEEE Trans Biomed Eng, , Vol. BME-14, No. 3, 1967, pp. 171-177.

[10] Snyder, M.F. and Rideout, V.C. (1969). Computer Simulation Studies of the Venous Circulation, IEEE Transactions on Bio-Medical Engineering,Vol.BME-16, No. 4, 1969, pp. 325-334.

[11] Liang, F.Y., Takagi, S., Himeno, R. and Liu, H. (2009). Biomechanical characterization of ventricular-arterial coupling during aging: A multi-scale model study. Journal of Biomechanics, 42:692-704.

[12] Formaggia, L., Quarteroni, A. and Veneziani, A. (2009). Cardiovascular Mathematics, Modeling and simulation of the circulatory system,SpringerVerlag Italia, Milano.

[13] Blanco, P.J. and Feij oo, R.A. (2010). A 3D-1D-0D Computational model for the entire cardiovascular system. Computational Mechanics, eds. E.Dvorking, M. Goldschmit, M. Storti, XXIX 5887-5911.

[14] Alastruey, J., Moore, S.M., Parker, K.H., David, T., Peir'o, J. and Sherwin S.J. (2008). Reduced modelling of blood flow in the cerebral circulation: Coupling 
1-D, 0-D and cerebral auto-regulation models. International journal for numerical methods in fluids, Vol. 56 no. 8, pp. 1061-1067.

[15] Nzerem, F. E. and Ugorji, H. C. (2019). Cardiac conduction system: the graph theoretic approach, J. Math. Comput. Sci., 9, pp.303-326.

[16] Von, B. L. (1950). The theory of open systems in physics and biology. Science 111, pp.23-29.

[17] Branko, F. (2015). The Heart: Pressure-Propulsion Pump or Organ of Impedance? Journal of Cardiothoracic and Vascular Anesthesia, Vol 29, No 6, pp 1688-1701.

[18] Nithiarasu, P., (2021). Biofluid Dynamics, https://www.maths.gla.ac.uk Accessed 14 June, 2021.

[19] Formaggia, L., Quarteroni, A. and Veneziani, A. (2009). Cardiovascular mathematics. Vol. 1. Springer, Heidelberg.

[20] Cani c, S and Kim, E.H. (2003). Mathematical analysis of the quasilinear effects in a hyperbolic model blood ow through compliant axi-symmetric vessels. Mathematical Methods in the Applied Sciences, 26, pp.1161-1186.

[21] Buxton, G.A. and Clarke, N. (2006). Computational phlebology:the simulation of a vein valve, Journal of Biological Physics, Vol. 32, no. 6, pp. 507-521.

[22] Larrabidea, I., Blanco, P.J., Urquiza, S.A., Dari, E.A., V'eneref, M.J., de Souza e Silvac, N.A. and Feij oo, R.A. (2012). HeMoLab -hemodynamics modelling laboratory: an application for modelling the human cardiovascular system. Computers inBiology and Medicine, Vol. 42, pp. 993-1004.

[23] Sherwin, S., Franke, V. Peir'o, and Parker, J. K. (2003). One-dimensional modelling of a vascular network in pace-time variables.Journal of Engineering Mathematics, Vol. 47, pp. 217.

[24] van de Vosse, F.N. and Stergiopulos, N. (2011). Pulse wave propagation in the arterial tree. Annual Review of Fluid Mechanics, Vol. 43, pp. 467-499.

[25] Bessonov, N., Sequeira, A., Simakov, S., Yu. V. and, Volpert, V. (2016). Methods of Blood Flow Modelling, Math. Model. Nat. Phenom.Vol. 11, No. 1, pp.1-25, DOI: 10.1051/mmnp/201611101. 